\title{
Annihilation-diffusion processes: an exactly solvable model
}

\author{
Farinaz Roshani ${ }^{a}$ and Mohammad Khorrami ${ }^{b}$ \\ Institute for Advanced Studies in Basic Sciences, \\ P. O. Box 159, Zanjan 45195, Iran \\ Institute for Studies in Theoretical physics and Mathematics, \\ P. O. Box 5531, Tehran 19395, Iran \\ ${ }^{a}$ roshani@iasbs.ac.ir \\ ${ }^{b}$ mamwad@iasbs.ac.ir
}

\begin{abstract}
A family of diffusion-annihilation processes is introduced, which is exactly solvable. This family contains parameters that control the diffusion- and annihilation- rates. The solution is based on the Bethe ansatz and using special boundary conditions to represent the reaction. The processes are investigated, both on the lattice and on the continuum. Special cases of this family of processes are the simple exclusion process and the drop-push model.
\end{abstract}

PACS numbers: 82.20.Mj, 02.50.Ga, 05.40.-a 


\section{Introduction}

In recent years, the asymmetric exclusion process and the problems related to it, including for example the bipolimerization [1], dynamical models of interface growth 2], traffic models [3], the noisy Burgers equation [4], and the study of shocks [5, [6], have been extensively studied. The dynamical properties of this model have been studied in [6-8]. As the results obtained by approaches like mean field are not reliable in one dimension, it is useful to introduce solvable models and analytic methods to extract exact physical results. Among these methodes is the coordinate Bethe-ansatz, which was used in [9] to solve the asymmetric simple exclusion process on a one-dimensional lattice. In [10], a similar technique was used to solve the drop-push model [11], and a generalized one-parameter model interpolating between the asymmetric simple exclusion model and the drop-push model. In [12], this family was further generalized to a family of processes with arbitrary left- and right- diffusion rates. All of these models were lattice models. Finally, the behaviour of latter model on continuum was investigated in [13]. The continuum models of this kind are also investigated in 14,15$]$.

In the generalized model interpolating between the asymmetric simple exclusion model and the drop-push model [10, 12, 13], there are two paprameters $\lambda$ and $\mu$, which control the pushing rate. Normalizing the diffusion rate to one, it is seen that the sum of these two parameters should be one to ensure the conseravtion of probability. These two parameters appear in the boundary condition used instead of the reaction. A question arises that what happens if one violates this conservation of probability. This is what is investigated in the present paper.

The scheme of the paper is the following. In section 2 , the allowed boundary conditions are investigated. It is shown that if $\lambda+\mu<1$, then the number of the particles will be decreasing, that is, there is an annihilation process as well. It is shown that one can in fact write a two-particle to one-particle annihilation process which results in such a boundary condition.

In section 3, the Bethe-ansatz solution for the $N$-particle probability of this process is obtained, and its large-time behaviour is investigated. This is done for the process on the lattice as well as on the continuum. Finally, in section 4 the special case of the two-particle initial condition is fully investigated, and it is explicitely shown that at large times, there remains only one particle.

\section{Boundary conditions}

Consider the following master equation for an asymmetric exclusion process.

$$
\begin{aligned}
\frac{\partial}{\partial t} P\left(x_{1}, x_{2}, \cdots, x_{N} ; t\right)= & P\left(x_{1}-1, x_{2}, \cdots, x_{N} ; t\right) \\
& +P\left(x_{1}, x_{2}-1, \cdots, x_{N} ; t\right)+\cdots
\end{aligned}
$$




$$
\begin{aligned}
& +P\left(x_{1}, x_{2}, \cdots, x_{N}-1 ; t\right) \\
& -N P\left(x_{1}, x_{2}, \cdots, x_{N} ; t\right) .
\end{aligned}
$$

This equation describes a collection of $N$ particles drifting to the right with unit rate. If the particles are to exclude each other, that is if no two particles are to occupy the same site, then (11) is valid only for

$$
x_{i}<x_{i+1}-1 .
$$

One can, however, assume that (11) is correct for all of the physical region $x_{i}<$ $x_{i+1}$, and impose certain boundary conditions for $x_{i}=x_{i+1}$. Note that if $x_{i}=x_{i+1}-1$ for some $i$, then in the right-hand side of (11) there will be terms with $x_{i}=x_{i+1}$, which is out of the physical region. The boundary condition determines the nature of the interaction between particles. But what are the allowed boundary conditions? Let's rewrite (11) for the case of two particles and use the conservation of probability. We arrive at

$$
\begin{aligned}
\frac{\partial}{\partial t} \sum_{x_{2}} \sum_{x_{1}<x_{2}} P\left(x_{1}, x_{2} ; t\right)= & \sum_{x_{2}} \sum_{x_{1}<x_{2}} P\left(x_{1}, x_{2} ; t\right) \\
& -\sum_{x} P(x, x+1 ; t) \\
& +\sum_{x_{2}} \sum_{x_{1}<x_{2}} P\left(x_{1}, x_{2} ; t\right) \\
& +\sum_{x} P(x, x ; t) \\
& -2 \sum_{x_{2}} \sum_{x_{1}<x_{2}} P\left(x_{1}, x_{2} ; t\right), \\
= & -\sum_{x} P(x, x+1 ; t)+\sum_{x} P(x, x ; t) .
\end{aligned}
$$

If the right-hand side of (3) is to be identical to zero, then $P(x, x)$ should be a linear combination of $P(x-1+i, x+i)$ 's. This may work for the two-particle process, but in many-partcles processes it may introduce terms with $x_{i}>x_{i+1}$, which need additional boundary conditions. The only exception is when $P(x, x)$ is a linear combination of only $P(x, x+1)$ and $P(x-1, x)$. So one can write

$$
P(x, x)=\lambda P(x, x+1)+\mu P(x-1, x) .
$$

Inserting this in (3), one arrives at

$$
\frac{\partial}{\partial t} \sum_{x_{2}} \sum_{x_{1}<x_{2}} P\left(x_{1}, x_{2} ; t\right)=(\lambda+\mu-1) \sum_{x} P(x, x+1 ; t) .
$$

In order that the right-hand side of (5) be zero, one must impose

$$
\lambda+\mu=1 .
$$


This is the boundary condition used in 10, 12, 13.

This kind of boundary condition ensures the conservation of particle number. But in a process where annihilation exists as well, the number of the particles is not conserved; it is decreasing. It is seen that if

$$
\lambda+\mu<1
$$

then the probability of finding two particles is decreasing. Suppose one begins with two particles. They drift to right with unit rate. If they meet each other, either the left particle is stopped, or one of them is annihilated. That is, we have the following processes:

$$
\begin{array}{ll}
A \emptyset \rightarrow \emptyset A, & \text { with rate } 1, \\
A A \rightarrow \emptyset A, & \text { with rate } \alpha, \\
A A \rightarrow A \emptyset, & \text { with rate } \beta
\end{array}
$$

In this case, still the more-than-two particle densitiies are zero, since no particles are generated during the process. But the two-particle density does not determine the one-particle density. And the summation of the former need not be a constant (one). It is seen that the master equation for the two-particle probability is

$$
\begin{array}{rlr}
\frac{\partial}{\partial t} P\left(x_{1}, x_{2} ; t\right)= & P\left(x_{1}-1, x_{2} ; t\right)+P\left(x_{1}, x_{2}-1 ; t\right) \\
& -2 P\left(x_{1}, x_{2} ; t\right), \quad x_{1}<x_{2}-1,
\end{array}
$$

and

$$
\frac{\partial}{\partial t} P(x, x+1 ; t)=P(x-1, x+1 ; t)-(1+\alpha+\beta) P(x, x+1 ; t) .
$$

But (10) is the same as (9), provided one uses the boundary condition

$$
P(x, x)=\lambda P(x, x+1),
$$

with

$$
\lambda=1-(\alpha+\beta) .
$$

So the difference $1-\lambda$ is in fact related to the annihilation rate, as expected. There is one other thing to be noted. As the number of particles is not conserved, one cannot calculate the one-particle probability by a simple summation of the two-particle probability. That is,

$$
P(x) \neq \sum_{y>x} P(x, y)+\sum_{y<x} P(y, x) .
$$

In fact, for the process described, the particles interact and annihilate each other, until there remains only one particle. This means that at $t \rightarrow \infty$, there 
will be only one particle. So the more-than-one-particle probabilities will tend to zero, whereas the summation of the one-particle probability tends to one. However, if the initial condition is that there are $N$ particles, one can write differential equations for $n$-particle probabilities in which $n$-particle probabilities and $n+1$-particle probabilities occur (if $n<N$ ). For $n>N$, the $n$-particle probability is identically zero, and the equation for the $N$-particle probability is closed. So, in principle, one can find the $N$-particle probability first and use this to find less-than- $N$-particle probabilities. To be specific, the evolution equation for the one-particle probability is

$$
\begin{aligned}
\frac{\partial}{\partial t} P(x ; t)= & P(x-1 ; t)[1-P(x ; t)]-P(x ; t)[1-P(x+1 ; t)] \\
& -\alpha P(x, x+1 ; t)-\beta P(x-1, x ; t) .
\end{aligned}
$$

\section{Bethe-ansatz solution for the $N$-particle prob- ability}

Consider the Master equation (11), with the boundary condition

$$
P(\cdots, x, x, \cdots)=\lambda P(\cdots, x, x+1, \cdots),
$$

where $\lambda<1$. Following [9, 10, 12, 13], one can obtain the conditional probability using the Bethe ansatz. Writing

$$
P(\mathbf{x} ; t)=e^{E t} \Psi(\mathbf{x}),
$$

and

$$
\Psi(\mathbf{x})=\sum_{\sigma} A_{\sigma} e^{i \sigma(\mathbf{p}) \cdot \mathbf{x}},
$$

where the summation runs over the elements of the permutation group, one arrives at

$$
E=-N+\sum_{j} e^{-i p_{j}}
$$

and

$$
A_{\sigma \sigma_{i}}=S\left[\sigma\left(p_{i}\right), \sigma\left(p_{i+1}\right)\right] A_{\sigma},
$$

where $\sigma$ is that permutation which only interchanges $p_{i}$ and $p_{i+1}$. One also finds that

$$
S_{j k}:=S\left(p_{j}, p_{k}\right)=-\frac{1-\lambda e^{i p_{k}}}{1-\lambda e^{i p_{j}}} .
$$

This is the same as what found in 10, 12] with $\mu=0$, and if one puts $\lambda=1$, the result of [9] is obtained. The conditional probability is thus written as

$$
P(\mathbf{x} ; t \mid \mathbf{y} ; 0)=\int \frac{d^{N} p}{(2 \pi)^{N}} \Psi_{\mathbf{p}}(\mathbf{x}) e^{E(\mathbf{p}) t-i \mathbf{p} \cdot \mathbf{y}}
$$


where $\Psi$ is defined as $(17)$ with $A_{\text {identity }}=1$. This looks like similar to what obtained in [9, 10, 12]. There is, however, a difference. As $\lambda<1$, there is no pole in $S$, and hence in $A$. So for large times, when the probability distribution becomes smooth and its Fourier-transform for large frequencies tends to vanish, one can put $p_{j}=0$ in $S$ as an approximation to arrive at

$$
S \approx-1
$$

and

$$
A_{\sigma} \approx(-1)^{\sigma}
$$

One can also approximate $E(\mathbf{x})$ as

$$
E(\mathbf{p}) \approx \sum_{j}\left(-i p_{j}-\frac{p_{j}^{2}}{2}\right)
$$

So, for large times,

$$
P(\mathbf{x} ; t \mid \mathbf{y} ; 0) \approx \frac{1}{(2 \pi t)^{N / 2}} \sum_{\sigma}(-1)^{\sigma} e^{-\sum_{j}\left[x_{j}-\sigma\left(y_{j}\right)-t\right]^{2} /(2 t)} .
$$

It is clearly seen that the integral of this distribution over the physical region tends to zero as $t \rightarrow \infty$. This should be the case, since the number of the particles does not remain constant and decreases.

Using the boundary condition (4) with (7), doesn't change the results drastically. In fact, $E$ doesn't change at all, while $S$ is changed to

$$
S_{j k}=-\frac{1-\lambda e^{i p_{k}}-\mu e^{-i p_{j}}}{1-\lambda e^{i p_{j}}-\mu e^{-i p_{k}}} .
$$

The approximate result for large times does not depend on $\lambda$ or $\mu$, so long as their sum is less than 1.

One can also investigate the continuous-space form of the evolution. Following [13], the master equation is changed to

$$
\frac{\partial}{\partial t} P(\mathbf{x} ; t)=-\sum_{j} \partial_{j} P(\mathbf{x} ; t)+\frac{1}{2} \sum_{j} \partial_{j}^{2} P(\mathbf{x} ; t),
$$

and the boundary condition to

$$
\left.\left(1-\lambda-\mu-\lambda \partial_{j+1}+\mu \partial_{j}\right) P\right|_{x_{j+1}=x_{j}}=0 .
$$

Using the Galilean transformation $x_{i} \rightarrow x_{i}+v t$ and $t \rightarrow t$, the master equation (27) is simplified to

$$
\frac{\partial}{\partial t} P(\mathbf{x} ; t)=\frac{1}{2} \nabla^{2} P(\mathbf{x} ; t)
$$


Using a Bethe-ansatz solution like (16) and (17), one arrives at

$$
E=-\frac{1}{2} \sum_{j} p_{j}^{2},
$$

and

$$
S_{j k}=-\frac{1-\lambda-\mu-i \lambda p_{k}+i \mu p_{j}}{1-\lambda-\mu-i \lambda p_{j}+i \mu p_{k}} .
$$

For large times, one can approximate $S$ to -1 , and arrive at a result similar to (25). The difference is that in the exponent the term $x_{j}-t-\sigma\left(y_{j}\right)$ is replaced by $x_{j}-\sigma\left(y_{j}\right)$, as the Galilean transformation used has canceled the drift from the master equation.

\section{Two-particle system and the exact solution}

As it was seen in the previous section, the conditional probability for the twoparticle system described by (9) and (11) is

$$
\begin{aligned}
P(\mathbf{x} ; t \mid \mathbf{y} ; 0)= & \int \frac{d^{2} p}{4 \pi^{2}} e^{E t-i \mathbf{p} \cdot \mathbf{y}} \\
& \times\left[e^{i\left(p_{1} x_{1}+p_{2} x_{2}\right)}-\frac{1-\lambda e^{i p_{2}}}{1-\lambda e^{i p_{1}}} e^{i\left(p_{1} x_{2}+p_{2} x_{1}\right)}\right],
\end{aligned}
$$

where $E$ is obtained from (18). This integration is easily done and the result is

$$
\begin{aligned}
P(\mathbf{x} ; t \mid \mathbf{y} ; 0)= & e^{-2 t} \frac{t^{x_{1}-y_{1}}}{\left(x_{1}-y_{1}\right) !} \frac{t^{x_{2}-y_{2}}}{\left(x_{2}-y_{2}\right) !} \\
& \left.+e^{-2 t} \sum_{l=0}^{\infty} \frac{t^{l+x_{2}-y_{1}}}{\left(l+x_{2}-y_{1}\right) !} \frac{t^{x_{1}-y_{2}}}{\left(x_{1}-y_{2}\right) !} \lambda^{l}\left(-1+\frac{\lambda t}{x_{1}-y_{2}+1}\right) 33\right)
\end{aligned}
$$

Another interesting quantity is the average number of the particles. This is equal to the summation of the one-particle probability:

$$
N(t):=\sum_{x} P(x ; t) .
$$

Using (14), one arrives at

$$
\begin{aligned}
\dot{N} & =-(\alpha+\beta) \sum_{x} P(x-1, x ; t) \\
& =-\frac{1-\lambda}{\lambda} \sum_{x} P(x, x ; t) .
\end{aligned}
$$


The right-hand side can be calculated using the Bethe-ansatz solution directly. Using (32), one has

$$
\begin{aligned}
\sum_{x} P(x, x ; t) & =\int \frac{d^{2} p}{2 \pi} \delta\left(p_{1}+p_{2}\right)\left[1+S\left(p_{1}, p_{2}\right)\right] e^{E t-i\left(p_{1} y_{1}+p_{2} y_{2}\right)} \\
& =\lambda \int \frac{d p}{2 \pi} e^{2 t(\cos p-1)+i p\left(y_{2}-y_{1}\right)} \frac{e^{-i p}-e^{i p}}{1-\lambda e^{i p}} \\
& =\lambda \int \frac{d p}{2 \pi} e^{2 t(\cos p-1)+i p\left(y_{2}-y_{1}\right)} \\
& \times \sum_{m=0}^{\infty} \lambda^{m}\left[e^{i(m-1) p}-e^{i(m+1) p}\right] \\
= & \lambda \sum_{m=0}^{\infty} e^{-2 t} \lambda^{m}\left[\mathrm{I}_{y_{2}-y_{1}+m-1}(2 t)-\mathrm{I}_{y_{2}-y_{1}+m+1}(2 t)\right] \\
= & \lambda \sum_{m=0}^{\infty} e^{-2 t} \lambda^{m} \frac{y_{2}-y_{1}+m}{t} \mathrm{I}_{y_{2}-y_{1}+m}(2 t)
\end{aligned}
$$

where I denotes the modified Bessel function. This can be inserted in (35) to obtain

$$
\begin{aligned}
N(t) & =N(0)-\frac{1-\lambda}{\lambda} \int_{0}^{t} d t^{\prime} \sum_{x} P\left(x, x ; t^{\prime}\right) \\
& =N(0)-(1-\lambda) \sum_{m=0}^{\infty} \int_{0}^{t} d t^{\prime} e^{-2 t^{\prime}} \lambda^{m} \frac{y_{2}-y_{1}+m}{t^{\prime}} \mathrm{I}_{y_{2}-y_{1}+m}\left(2 t^{\prime}\right) .
\end{aligned}
$$

This is simplified for $t \rightarrow \infty$. Using

$$
\int_{0}^{\infty} d s \frac{e^{-s}}{s} \mathrm{I}_{n}(s)=\frac{1}{n},
$$

one arrives at

$$
\begin{aligned}
N(\infty) & =N(0)-(1-\lambda) \sum_{m=0}^{\infty} \lambda^{m} \\
& =N(0)-1
\end{aligned}
$$

But note that

$$
N(0)=2
$$

since at the beginning there were two particles at $y_{1}$ and $y_{2}$. That is,

$$
P(x ; 0)=\delta\left(x-y_{1}\right)+\delta\left(x-y_{2}\right) .
$$

So, at $t \rightarrow \infty$, there remains only one particle, as one of the two particles has been annihilated. 
The continuous-space analogue of this model can also be solved easily. Using (29) as the master equation, and (30) and (31) (with $\mu=0$ ), one is led to

$$
\begin{aligned}
P(\mathbf{x} ; t \mid \mathbf{y} ; 0)= & \int \frac{d^{2} p}{4 \pi^{2}} e^{E t-i \mathbf{p} \cdot \mathbf{y}} \\
& \times\left[e^{i\left(p_{1} x_{1}+p_{2} x_{2}\right)}-\frac{1-\lambda-i \lambda p_{2}}{1-\lambda-i \lambda p_{1}} e^{i\left(p_{1} x_{2}+p_{2} x_{1}\right)}\right] .
\end{aligned}
$$

Using the change of variable $p:=p_{1}+i(1-\lambda) / \lambda$ in the second integral, (42) is written as

$$
\begin{aligned}
P(\mathbf{x} ; t \mid \mathbf{y} ; 0)= & \frac{1}{2 \pi t} e^{-\left[\left(x_{1}-y_{1}\right)^{2}+\left(x_{2}-y_{2}\right)^{2}\right] /(2 t)} \\
& +\frac{1}{\sqrt{8 \pi t}}\left[A+\frac{x_{1}-y_{2}}{t}\right] e^{-\left(x_{1}-y_{2}\right)^{2} /(2 t)} e^{\left[2 A\left(x_{2}-y_{1}\right)+t A^{2}\right] / 2} \\
& \times\left\{-1+\operatorname{erf}\left[\frac{1}{\sqrt{2 t}}\left(x_{2}-y_{1}+t A\right)\right]\right\},
\end{aligned}
$$

where

$$
A:=\frac{1-\lambda}{\lambda} .
$$

One notes that at $t \rightarrow \infty$, the conditional probability is simplified. We have

$$
1-\operatorname{erf}(x) \approx \frac{e^{-x^{2}}}{x \sqrt{\pi}}, \quad \text { for large } x
$$

From this, it is seen that at $t \rightarrow \infty$

$$
P(\mathbf{x} ; t \mid \mathbf{y} ; 0) \approx \frac{1}{2 \pi t}\left\{e^{-\left[\left(x_{1}-y_{1}\right)^{2}+\left(x_{2}-y_{2}\right)^{2}\right] /(2 t)}-e^{-\left[\left(x_{1}-y_{2}\right)^{2}+\left(x_{2}-y_{1}\right)^{2}\right] /(2 t)}\right\} .
$$

This is a special case of what obtained in the previous section.

Another quantity to be considered is the one-point probability. In the continuum limit, and after performing the Galilean transformation, (14) becomes

$$
\frac{\partial}{\partial t} P(x ; t)=\frac{1}{2} \frac{\partial^{2}}{\partial x^{2}} P(x ; t)-\left[\alpha\left(1+\partial_{2}\right)+\beta\left(1-\partial_{1}\right)\right] P(x, x ; t) .
$$

From this, using the boundary condition (28), with $\mu=0$, one arrives at

$$
\dot{N}(t)=-\frac{1-\lambda}{\lambda} \int d x P(x, x ; t),
$$

where

$$
N(t):=\int d x P(x ; t)
$$


Using (42), the integral at the right-hand side is calculated to be

$$
\int d x P(x, x ; t)=\int \frac{d p}{2 \pi} \frac{-2 i \lambda p}{1-\lambda-i \lambda p} e^{-t p^{2}+i p\left(y_{2}-y_{1}\right)} .
$$

To obtain $N(\infty)$, one integrates (50) from 0 to $\infty$. This results in

$$
\begin{aligned}
\int_{0}^{\infty} d t \int d x P(x, x ; t) & =\frac{\lambda}{\pi} \mathrm{P} \int \frac{d p}{i p} \frac{e^{i p\left(y_{2}-y_{1}\right)}}{1-\lambda-i \lambda p} \\
& =\frac{\lambda}{1-\lambda} .
\end{aligned}
$$

The symbol $\mathrm{P}$ denotes the Cauchy's principle value, and use has been made of the fact that $y_{2}>y_{1}$. From this, it is found that

$$
\begin{aligned}
N(\infty) & =N(0)-1 \\
& =1
\end{aligned}
$$

This is the same result obtained for the lattice, as expected. 


\section{References}

[1] C. T. MacDonald, J. H. Gibbs, \& A. C. Pipkin; Biopolimers 6 (1968) 1.

[2] J. Krug \& H. Spohn; in Solids far from equilibrium, edited by C. Godreche (Cambridge University Press, Cambridge, England, 1991), and references therein.

[3] K. Nagel; Phys. Rev. E53 (1996) 4655.

[4] J. M. Burgers, The nonlinear diffusion equation (Reidel, Boston, 1974).

[5] B. Derrida, S. A. Janowsky, J. L. Lebowitz, \& E. R. Speer; Europhys. Lett. 22 (1993) 651.

[6] P. A. Ferrari \& L. R. G. Fontes; Probab. Theory Relat. Fields 99 (1994) 305.

[7] T. Ligget, Interacting partcle systems (Springer Verlag, Newyork, 1985).

[8] L. H. Gava \& H. Spohn; Phys. Rev. A46 (1992) 844.

[9] G. M. Schütz; J. Stat. Phys. 88 (1997) 427.

[10] M. Alimohammadi, V. Karimipour, \& M. Khorrami; Phys. Rev. E57 (1998) 6370.

[11] G. M. Schütz \& E. Domnay; J. Stat. Phys. 72 (1993) 277.

[12] M. Alimohammadi, V. Karimipour, \& M. Khorrami; J. Stat. Phys. 97 (1999) 373.

[13] F. Roshani \& M. Khorrami; Phys. Rev. E60 (1999) 3393.

[14] T. Sasamoto \& M. Wadati; J. Phys. Soc. Japan E67 (1998) 784.

[15] T. Sasamoto \& M. Wadati; J. Phys. A31 (1998) 6057. 